Original Article

\title{
The immediate effects of proprioceptive neuromuscular facilitation with taping on gait parameters in patients with chronic stroke
}

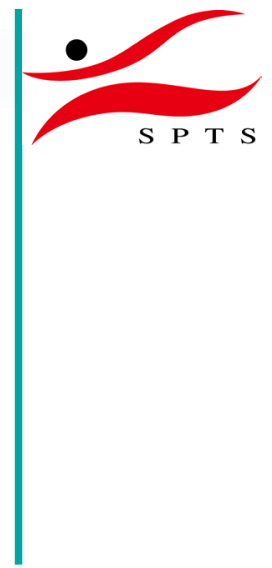

\author{
SHIN-Jun PARK, PT, MS ${ }^{1)}$ \\ 1) Department of Physical Therapy, Gangdong College: 278 Daehak-gil, Danpyeong-ri, \\ Gamgok-Myeon, Eumseong-gun, Chungcheongbuk-do, Republic of Korea
}

\begin{abstract}
Purpose] The purpose of the present study was to examine the immediate effects of simultaneous application of proprioceptive neuromuscular facilitation (PNF) and elastic taping (T), and the removal of the elastic tape, on the gait parameters of stroke patients. [Subjects and Methods] Twenty stroke patients were divided into a proprioceptive neuromuscular facilitation group (PNFG, $n=7)$, a taping group $(\mathrm{TG}, \mathrm{n}=6)$, and a proprioceptive neuromuscular facilitation with taping group (PNFTG, $n=7$ ). Relevant interventions were applied for 30 minutes, the tape was removed, and gait parameters were evaluated. [Results] Only the intervention of the PNFTG yielded significant differences in patient cadence, speed, and stride length. [Conclusion] The simultaneous application of PNF and taping for 30 minutes has carryover effects that can improve stroke patients' gait ability, even after the removal of the tape.

Key words: Proprioceptive neuromuscular facilitation, Taping, Stroke
\end{abstract}

(This article was submitted Aug. 14, 2017, and was accepted Aug. 26, 2017)

\section{INTRODUCTION}

In stroke patients, the gait pattern is asymmetric and their gait speed is reduced ${ }^{1,2)}$. Increased gait speed in stroke patients leads to better function and quality of life ${ }^{3}$. Since the rehabilitation of stroke patients has primarily focused on the improvement and maintenance of gait thus far ${ }^{4}$, physical therapists should consider developing new intervention methods that are required by patients and that can effectively improve the gait ability in a short period of time.

Taping ${ }^{5)}$, electrotherapy ${ }^{6}$ and proprioceptive neuromuscular facilitation (PNF) methods ${ }^{7}$ have been applied to improve the immediate walking ability of stroke patients. Among these, taping around the knee joint has been reported to have an immediate effect on improving the walking speed of stroke patients ${ }^{5)}$. Although taping has the advantages of enabling protector education, because of its simple attaching methods, and allows for the cutting of the tape to fit the body, it also has problems extending its effects, as carry-over effects cannot be expected when the tape is removed after a single taping application ${ }^{8}$.

In addition, PNF is an intervention method that standardizes body and limb movements into patterns that can immediately improve stroke patients' gait speed ${ }^{7}$. When combined with taping, its effects have not only improved gait speed but also balance ability ${ }^{9)}$. However, previous studies conducted thus far have identified the improvement of gait speed after PNF application in acute stroke patients, but not in chronic stroke patients ${ }^{7)}$, and even those studies that applied a combination of taping and PNF to chronic stroke patients and identified the improvement of gait ability ${ }^{9}$ had limitations in failed to eliminate interference from other treatments because the intervention was applied for four weeks.

The taping with compression applied to the cerebral palsy continued for twenty minutes after the taping was removed ${ }^{8)}$. However, there is no study on the persistence of effects after removing taping from stroke patients.

Our study intends to examine stroke patients' gait ability in order to identify the effects appearing after a single PNF

Corresponding author. Shin-Jun Park (E-mail: 3178310@naver.com)

(C2017 The Society of Physical Therapy Science. Published by IPEC Inc.

(c) (1) $($ This is an open-access article distributed under the terms of the Creative Commons Attribution Non-Commercial No Derivatives

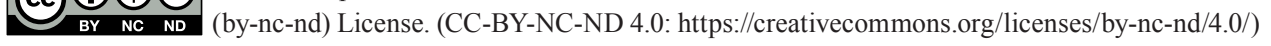


application, single taping application, and PNF combined with taping application and removal of the tape. Therefore, our study aimed to identify whether immediate improvement of gait ability appears after the taping intervention in stroke patients in whom PNF combined with taping is applied and the tape is removed thereafter, in order to examine the carry-over effects of the taping intervention.

\section{SUBJECTS AND METHODS}

The present study involved 20 hemiplegia patients in A rehabilitation hospital and B convalescent hospital located in Gyeonggi-do. The subjects were selected from among those who had been diagnosed with stroke at least six months earlier, who could independently walk at least $8 \mathrm{~m}$, understand explanations about the study process was going to work, had the result of paretic side ankle dorsiflexor manual muscle testing not lower than fair, had 3 or above on the Brunnstrom recovery stage 3, had no orthopedic disease of the ankle, had not undergone ankle surgery, and had no cutaneous allergy. The present study was conducted entirely in accordance with the Declaration of Helsinki, and all the subjects voluntarily participated in the study after understanding the purpose and intent of the study and signing a written agreement.

The subjects were divided into three groups, a PNFG group $(n=7)$, a taping group $(T G, n=6)$ and a proprioceptive neuromuscular facilitation with kinesio taping group (PNFTG, $n=7$ ). The individuals in the PNFG comprised five males and two females, aged $57.29 \pm 9.41$ years, with a height of $164.71 \pm 6.73 \mathrm{~cm}$, weight of $66.43 \pm 7.41 \mathrm{~kg}$, and a time since stroke of $24.86 \pm 7.38$ months. The individuals in the TG comprised four males and two females, aged $64.83 \pm 15.22$ years, with a height of $165.17 \pm 11.23 \mathrm{~cm}$, weight of $60.17 \pm 12.89 \mathrm{~kg}$, and a time since stroke of $25.00 \pm 5.40$ months. The individuals in the PNFTG comprised seven males, aged $51.71 \pm 6.47$ years, with a height of $171.29 \pm 5.65 \mathrm{~cm}$, weight of $71.43 \pm 12.20 \mathrm{~kg}$, and a time since stroke of $33.43 \pm 9.14$ months.

Gait parameter was measured using a BTS G-Walker (Bioengineering S.p.A., Milan, Italy). The parameters measured during subjects' gaits were the cadence, speed, paretic side double support duration, and stride length. For the evaluation, a G-sensor was fixed to L5 of the subject, and the subject was directed to walk straight on an 8 m-long walkway located in front, at a comfortable pace ${ }^{10)}$.

All the subjects rested for 10 minutes prior to the initial measurement, and the evaluation was conducted after applying PNF for 30 minutes in the case of the PNFG, after removing the tape following a rest for 30 minutes with the tape remaining attached in the case of the TG, and after removing the tape immediately after simultaneous application of taping and PNF for 30 minutes in the case of the PNFTG. All the study interventions were performed by an experienced physiotherapist who completed PNF level 1 and 2 courses.

The PNF was applied for 30 minutes with a combination of the sprinter pattern and skater pattern in a sitting position or half-standing position. The sprinter pattern consisted of alternating applications of flexion-adduction-external rotation of the non-paretic upper extremities, and flexion-abduction-internal rotation of the paretic lower extremities. The skater pattern consisted of alternating applications of extension-adduction-internal rotation of the non-paretic upper extremities, and extension-adduction-external rotation of the paretic lower extremities ${ }^{11)}$.

The taping intervention was implemented using $5 \mathrm{~cm}$-wide adhesive elastic tapes (Kinesiology $3 \mathrm{NS}$ Tape, TS Co., Ltd., Seoul, Korea). The tapes were attached to the quadriceps muscles ${ }^{12)}$ and tibialis anterior muscle ${ }^{13)}$ in the directions of the paretic side muscles. First, the tapes were cut based on the origins and insertion parts of the subjects' muscles. Then, the cut lengths of the tapes were folded into four equal parts to remove one-fourth of the tapes, and the remaining three-fourth lengths of tapes were stretched back to fit the body lengths.

All the data were processed and analyzed using the statistical program SPSS 20.0 (Windows ver.; IBM Corp., Armonk, NY, USA). The general characteristics of the subjects were indicated with descriptive statistics. In the study group, Wilcoxon signed-rank tests compared the results obtained before and after the intervention, the Kruskal-Wallis test was used to compare differences among the three groups, and Mann-Whitney $U$ tests were conducted in cases in which there were significant differences among the study groups after grouping the study groups into pairs (1-2, 2-3, 1-3). The significance level alpha $(\alpha)$ was 0.05

\section{RESULTS}

According to the results of the study, there were no significant differences in gait parameters observed in the PNFG and the TG conditions ( $p>0.05)$. PNFTG increased significantly in cadence, speed, and paretic side stride length $(\mathrm{p}<0.05)$. However, there was no significant difference among the three groups $(\mathrm{p}>0.05)$ (Table 1).

\section{DISCUSSION}

The PNF applied to acute stroke patients were immediate effects on gait speed, and long-term intervention brought about a further increase in gait speed ${ }^{7}$. However, the PNF applied to chronic stroke patients hardly were immediate improvement ${ }^{7)}$, and this was also the case with the PNFG in the present study. Although the application of spiral wound taping around chronic stroke patients' knees were immediate effects on gait ability in a study, its results, where the effects were measured when the 
Table 1. Comparison of gait parameter on each intervention

\begin{tabular}{|c|c|c|c|c|c|c|}
\hline \multicolumn{2}{|l|}{ Variable } & PNFG (n=7) & TG $(n=6)$ & PNFTG $(n=7)$ & $\chi^{2}$ & Post-hoc \\
\hline \multirow[t]{2}{*}{ Cadence } & Pre & $80.53 \pm 13.79$ & $98.35 \pm 8.94$ & $79.16 \pm 10.05$ & 3.893 & \\
\hline & Post & $86.74 \pm 14.14$ & $97.78 \pm 9.48$ & $87.63 \pm 8.05^{*}$ & & \\
\hline \multirow[t]{2}{*}{ Speed } & Pre & $0.79 \pm 0.20$ & $1.00 \pm 0.25$ & $0.84 \pm 0.23$ & 5.178 & \\
\hline & Post & $0.82 \pm 0.23$ & $0.98 \pm 9.48$ & $1.02 \pm 0.30^{*}$ & & \\
\hline \multirow[t]{2}{*}{ Stride length } & Pre & $1.21 \pm 0.21$ & $1.27 \pm 0.25$ & $1.39 \pm 0.38$ & 4.771 & \\
\hline & Post & $1.17 \pm 0.21$ & $1.28 \pm 0.24$ & $1.52 \pm 0.30^{*}$ & & \\
\hline \multirow[t]{2}{*}{ Double support duration } & Pre & $12.61 \pm 6.44$ & $12.65 \pm 4.45$ & $13.75 \pm 4.90$ & 0.308 & \\
\hline & Post & $13.29 \pm 5.97$ & $13.50 \pm 3.32$ & $14.76 \pm 7.28$ & & \\
\hline
\end{tabular}

Values are the mean and standard deviation, *Significant difference between before and after intervention in each group $(\mathrm{p}<0.05)$, PNFG: proprioceptive neuromuscular facilitation group; TG: taping group; PNFTG: proprioceptive neuromuscular facilitation with taping group

tape was attached, cannot be directly compared with the results of the present study, where the effects were evaluated after removing the tape ${ }^{5}$. In a study in which body functions were checked after removing the tape, no carryover effect appeared when the tape was removed in healthy persons ${ }^{14)}$, or cerebral palsy ${ }^{8)}$ patients. In the present study as well, no significant difference appeared following removal of the tape in the TG.

However, when palmar pressure was applied to the thenar of cerebral palsy children simultaneously with tape, and the tape was removed thereafter, carryover effects appeared with respect to hand functions 20 minutes later ${ }^{8}$. The PNFTG in the present study also were significant increases in the subjects' cadence and speed after removal of the tape. In a study of immediate effects of electric stimulation and exercise therapy on stroke patients, more significant differences appeared in $10 \mathrm{~m}$ gait following the application of a combination of manual exercise and electric stimulation than following the single applications of manual exercise or electric stimulation, supporting the results of the present study ${ }^{6}$. Although PNF does not have any immediate effect on chronic stroke patients without natural functional recovery, the method that assisted and promoted movements with simultaneous application of taping seems to were immediate effects for the improvement of stroke patients' gait speed. Because the reduction of gait speed of the stroke patients is represented by the weekness of the dorsi flexor $^{2)}$ and the knee flexor ${ }^{1)}$, the muscles of the taping facilitation method attached to this study were tibialis anterior and quadriceps muscles. However, there was no difference in the double support duration of the paretic side in this study. The decrease in walking speed of patients with stroke is due to various causes such as hip flexor weakness ${ }^{1)}$ and ankle spasticity ${ }^{2)}$.

In a previous study, PNF training and taping application for four weeks were significant improvement in 10-m gait and the Berg Balance Scale score of stroke patients ${ }^{9}$. However, since the 10-m gait measured in the previous study was applied using the stopwatch, only the gait speed can be identified. The gait evaluation measured in this study was conducted using equipment embedded with a triaxial accelerometer, magnetometer, and gyroscope, which is capable of evaluating spatiotemporal parameters of gait and thus, had an advantage of enabling the identification of diverse gait parameters.

Our study had several limitations. The number of subjects included was small, and it could be seen which groups the patients belonged to after randomization because of the physical characteristics that must enable independent gaits and the study design for taping attachment and exercise. In addition, since it was conducted by a specific institution, it could not be generalized to all stroke patients. However, if taping is combined with exercise, the fact that effects were measured after removing the tape is still meaningful. It is expected that more accurate results can be obtained if diverse study subjects are recruited and blind tests are performed. In addition, it will be even more meaningful to know when the gait speed lasts for a while, rather than the immediate effect.

\section{REFERENCES}

1) Hsu AL, Tang PF, Jan MH: Analysis of impairments influencing gait velocity and asymmetry of hemiplegic patients after mild to moderate stroke. Arch Phys Med Rehabil, 2003, 84: 1185-1193. [Medline] [CrossRef]

2) Lin PY, Yang YR, Cheng SJ, et al.: The relation between ankle impairments and gait velocity and symmetry in people with stroke. Arch Phys Med Rehabil, 2006, 87: 562-568. [Medline] [CrossRef]

3) Schmid A, Duncan PW, Studenski S, et al.: Improvements in speed-based gait classifications are meaningful. Stroke, 2007, 38: 2096-2100. [Medline] [CrossRef]

4) Dickstein R: Rehabilitation of gait speed after stroke: a critical review of intervention approaches. Neurorehabil Neural Repair, 2008, 22: 649-660. [Medline] [CrossRef]

5) Kim DD, Park SJ: The immediate effects of spiral taping on improvement of gait ability in patients with chronic stroke. J Digit Convergence, $2017,15: 529-536$. [CrossRef]

6) Yamaguchi T, Tanabe S, Muraoka Y, et al.: Immediate effects of electrical stimulation combined with passive locomotion-like movement on gait velocity and spasticity in persons with hemiparetic stroke: a randomized controlled study. Clin Rehabil, 2012, 26: 619-628. [Medline] [CrossRef]

7) Wang RY: Effect of proprioceptive neuromuscular facilitation on the gait of patients with hemiplegia of long and short duration. Phys Ther, 1994, 74: 1108- 
1115. [Medline] [CrossRef]

8) Keklicek H, Uygur F, Yakut Y: Effects of taping the hand in children with cerebral palsy. J Hand Ther, 2015, 28: 27-32, quiz 33. [Medline] [CrossRef]

9) Choi YK, Nam CW, Lee JH, et al.: The effects of taping prior to PNF treatment on lower extremity proprioception of hemiplegic patients. J Phys Ther Sci, 2013, 25: 1119-1122. [Medline] [CrossRef]

10) Pau M, Leban B, Collu G, et al.: Effect of light and vigorous physical activity on balance and gait of older adults. Arch Gerontol Geriatr, $2014,59: 568-573$. [Medline] [CrossRef]

11) Dietz B, Kim T, Lang E, et al.: Let's sprint, let's skate. Innovationen im PNF-Konzept. Springer, 2009, pp 44-45.

12) Kim EJ, Lee KB: Effects of kinesio taping to the quadriceps femoris muscles on functions of elderly women. J Phys Ther Sci, 2017, 29: 1205-1207. [Medline] [CrossRef]

13) Koseoglu BF, Dogan A, Tatli HU, et al.: Can kinesio tape be used as an ankle training method in the rehabilitation of the stroke patients? Complement Ther Clin Pract, 2017, 27: 46-51. [Medline] [CrossRef]

14) Zübeyir S, Nilüfer K, Burcu C, et al.: The effect of kinesiology taping on respiratory muscle strength. J Phys Ther Sci, 2012, 24: 241-244. [CrossRef] 